\title{
Characteristics and Prediction of Lung Cancer Mortality in China from 1991 to 2013
}

\author{
Jia-Ying Fang, Hong-Li Dong, Ku-Sheng Wu, Pei-Ling Du, Zhen-Xi Xu, Kun Lin*
}

\begin{abstract}
Objective: To describe and analyze the epidemiological characteristics of lung cancer mortality in China from 1991 to 2013, forecast the future five-year trend and provide scientific evidence for prevention and management of lung cancer. Materials and Methods: Mortality data for lung cancer in China from 1991 to 2013 were used to describe epidemiological characteristics. Trend surface analysis was applied to analyze the geographical distribution of lung cancer. Four models, curve estimation, time series modeling, gray modeling (GM) and joinpoint regression, were performed to forecast the trend for the future. Results: Since 1991 the mortality rate of lung cancer increased yearly. The rate for males was higher than that for females and rates in urban areas were higher than in rural areas. In addition, our results showed that the trend will continue to increase in the ensuing 5 years. The mortality rate increased from age 45-50 and peaked in the group of 85 years old. Geographical analysis indicated that people living in northeast China provinces and the coastal provinces in eastern China had a higher mortality rate for lung cancer than those living in the centre or western Chinese provinces. Conclusions: The standardized mortality rate of lung cancer has constantly increased from 1991 to 2013, and been predicted to continue in the ensuing 5 years. Further efforts should be concentrated on education of the general public to increase prevention and early detection. Much better prevention and management is needed in high mortality areas (northeastern and eastern parts of China) and high risk populations (45-50-year-olds).
\end{abstract}

Keywords: Lung cancer - epidemiological characteristics - standardized mortality - geographic distribution - prediction

Asian Pac J Cancer Prev, 16 (14), 5829-5834

\section{Introduction}

Lung cancer accounts for the largest number of cancerrelated deaths in China and throughout the world (World Health Organization, 2012). According to statistics from the Chinese National Cancer Registry, lung cancer was the leading cause of cancer deaths among people with malignant tumors in 2011, and the mortality rates of lung cancer in both urban and rural Chinese populations are higher than the worldwide average (Chen et al., 2015). The World Health Organization estimates that the annual lung cancer mortality rate in China may reach 1 million by 2025 .

The national cancer registration system makes it possible to study many aspects of lung cancer and provide available clues for national policy on cancer control. In addition, the regional data collection systems provide comparable data by sex or by area within a given period (Chen and Zhang, 2011). However, timely and accurate data on cancer are essential for effective lung cancer control. In addition, an accurate knowledge of lung cancer patterns and their development trend in the future are essential in the planning of national cancer control programs (Wei et al., 2012). In this paper, we use monitoring data of cancer mortality rate in China for the period 1991-2013 to describe the dynamics of death, sex, age, urban-rural location, and geographic distribution of lung cancer to determine lung cancer prevention strategies in China. Prediction of the trends and identification of geographic patterns of lung cancer mortality, based on population and location, may provide important clues regarding the cause and effect of the relevant factors. Therefore, we used trend surface analysis, one of the most widely used to reflect geographical distribution and trends of disease, to describe the geographical distribution of lung cancer mortality rate in China.

\section{Materials and Methods}

\section{Data Source}

Lung cancer mortality rate data in 1991-2013 and Cancer mortality rates, by sex, age group and urban/rural 
residence, came from the nation-wide cancer mortality survey of the Disease Prevention and Control Bureau, Ministry of Public Health (Chen, 2008; Zhao, 2008; Zhao, 2009; Zhao, 2010; He, 2011; He, 2012). Demographic data was collected from the Chinese National Statistics Department. The National Central Cancer Registry of China collected cancer mortality data from populationbased cancer registries in China which included 38 monitoring sites (17 in the urban area and 21 in the rural area). Data collection followed the rules and standards of the International Agency for Research on Cancer (IARC) and the International Association for Cancer Registration (China health statistics yearbook, 2005; China health statistics yearbook, 2006; China health statistics yearbook, 2007; China health statistics yearbook, 2008; Chen et al., 2013; Chen et al., 2014; China health statistics yearbook, 2014; Chen et al., 2015).

Three aspects were used to evaluate the quality of the data including diagnosis reliability, data integrity and coding quality. National Central Cancer Registry (NCCR) was responsible for collecting data, calculating the statistical items accurately, analyzing the data of cancer incident cases and deaths from registration areas, and then releasing the updated data in Cancer Registry Annual Report. To ensure reliability, the quality of data was assessed based on "Guideline of Chinese Cancer Registration" and referred to the criteria for "Cancer Incidence in Five Continents Volume IX" by the International Agency for Cancer Registry (IACR), the International Agency for Research on Cancer (IARC). Database softwares, such as MS-FoxPro, MS-Excel, SAS and IARC issued by IARC/IACR were used for data collection sorting, check and evaluation (Chen et al., 2013).

\section{Statistical analysis}

The crude rate $(\mathrm{CR})$, standardized mortality rate (SMR), age-standardized rate by Chinese standard population (CASR) were used in the statistical analysis. Chinese population census in 1982 and Segi's population were used for age-standardized incidence/mortality rates.

\section{Methods}

Cancer mortality data from 1991-2013 was used to evaluate the dynamic changes in SMR of lung cancer, identify the change of distributions by population, time and place, and estimate the trend over the next 5 years.

The Trend-surface Analysis method was used to study the geographic distribution of lung cancer mortality in China. This global surface-fitting method generates a three-dimensional surface which depicts a major spatial trend of lung cancer mortality, and geographical anomalies could be highlighted in this way using SAS 9.2. Polynomial regression was applied to fit in a trend surface analysis based on standardized mortality of longitude (x) and latitude (y), and the SMR of various areas. Four aspects --- the R-square test, F-test, goodness of fit order by order test, and a subjective judgment method were applied to select the order of trend surface modeling (Shi et al., 2014).

Four different methods --- curve estimation, time series modeling, gray modeling (GM) and Joinpoint regression were used to predict the lung cancer mortality trends in the next 5-year. Statistical analysis was performed by SPSS19.0, DPS 9.50 and Joinpoint 3.3.5.

The curve estimation models including linear, logarithmic, inverse, quadratic, cubic, power, compound, S-curve, logistic, growth, and exponential are types of linear and nonlinear curves. SPSS software was used to assess which type of curve best estimates the potential trend distribution of lung cancer data. In this investigation, $\mathrm{x}$ stands for the time (year) and y stands for standardized mortality rates (SMR).

The time series models try to predict epidemiological behaviors by previously observed values. Many researchers have applied different time series models to forecasting epidemic incidence or mortality in previous studies (Li et al., 2012; Spaeder and Fackler, 2012). The autoregressive integrated moving average (ARIMA) models are almost the most widely used methods, considering both the historical values and residuals, and can effectively transforms the non-stationary data into a stationary one (Zhang et al., 2014).

Gray system theory uses a black-gray-white color spectrum to describe a complex system. A white color denotes a system that is completely characterized and a black color represents a system that is totally unknown (Huang et al., 2013). GM $(1,1)$ means a gray model with first order differential time series equations in one variable which is the most widely used dynamic model of the gray system theory and can be used for the prediction of the dominant factor in the complex thing (Shi et al., 2014). Based on the theory of the gray model, X (i) means the standardized mortality rate of lung cancer, $t$ means the year. This paper used the improved GM $(1,1)$ model to predict the change of lung cancer in China.

Joinpoint regression model was applied to analyze the long-term trends of lung cancer mortality. In Joinpoint regression model, dependent variable $\mathrm{x}$ is the year, and independent variable $y$ is the mortality rate. The models incorporated both the estimated the annual percentage rate change and average annual percent change of lung cancer in China as well as the standard error of the estimated rates and can test whether a multi-segmented line fits the data better than a straight line (Qiu et al., 2009).

\section{Results}

Time-dependent changes in Mortality Rates of lung cancer Data from three national surveys on lung cancer mortality during the periods of 1973-1975, 1990-1992, and 2004-2005 showed that the mortality rates of lung cancer were $5.46 / 10^{5}$ in $1973-1975,17.54 / 10^{5}$ in 1990 1992 , and $30.83 / 10^{5}$ in $2004-2005$, respectively. The age-standardized rate by China's population of 1982 was $5.60 / 10^{5}, 15.19 / 10^{5}$ and $20.24 / 10^{5}$ respectively. The standardized mortality of lung cancer increased steadily from 1973 to 2009 , with lung cancer mortality being $49.44 / 10^{5}$ in 2011 , the standardized mortality rate $34.95 / 10^{5}$. The control strategies might be further emphasized in the future for the effective prevention of lung cancer in China (Chen et al., 2015). 
Difference between the urban and rural lung cancer mortality

The age-specific mortality in rural areas was lower than that in urban areas in most of age groups. The rural lung cancer mortality was 37.44 per 100,000 while in urban areas it was 46.40 per 100,000. After adjusting the age, mortality in urban areas was still significantly higher than the rural areas. The mortality rate in urban areas peaked at 484.36 per 100,000 in 85 year-old age group, while rural lung cancer mortality peaked at 345.75 per 100,000 in the $80 \sim 84$ age group, being earlier than urban areas (Figure 1). The standardized mortality increased in both rural and urban areas, and lung cancer mortality being stabilized

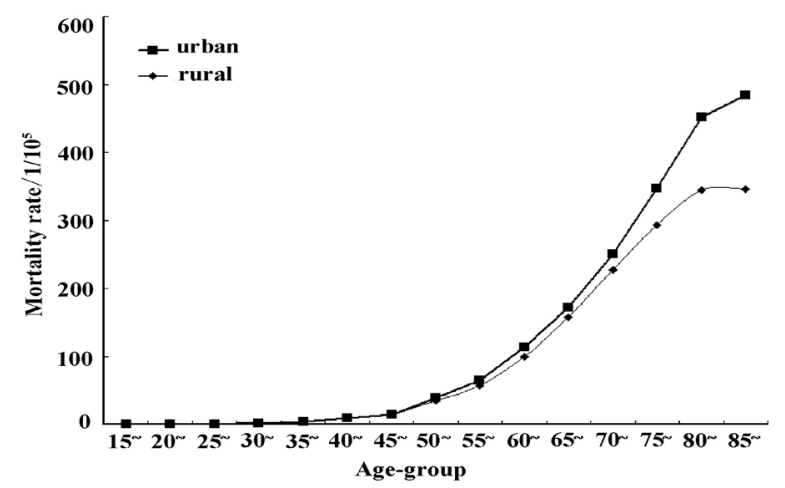

Figure 1. Change in Age-Specific Mortality of Lung Cancer between Urban and Rural Areas in Change in Age-Specific Mortality of Lung Cancer between Urban and Rural Areas in China in 2013

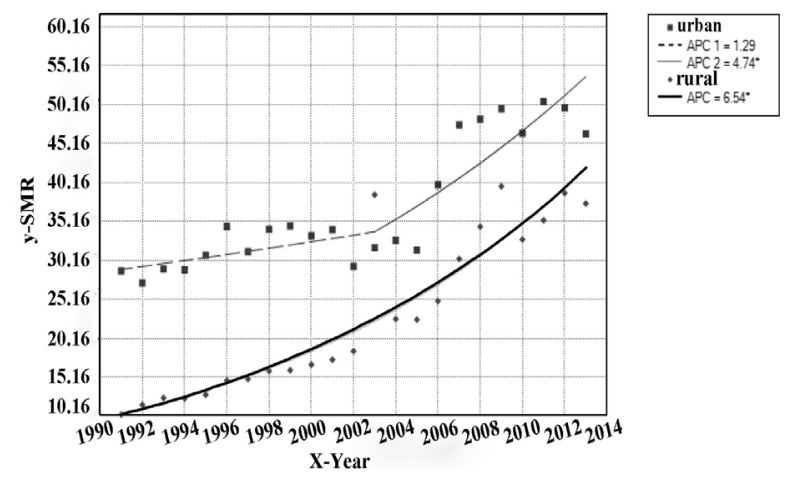

Figure 2. Trends in the Mortality of Lung Cancer by Joinpoint Analysis by in China from 1991-2013 (1/10 $\left.{ }^{5}\right)$

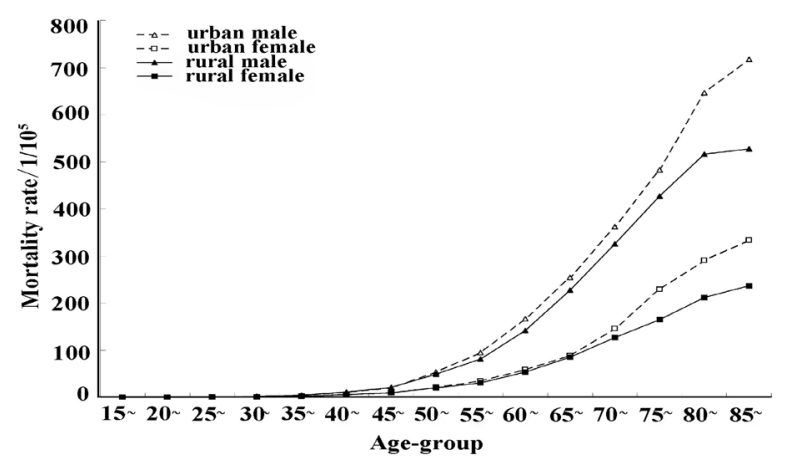

Figure 3. Change in Age-Specific Mortality of Lung Cancer among Urban and Rural Areas, and Different Sex in China in 2013 at high levels in rural areas while being volatile in rural areas (Figure 2). With Joinpoint regression analysis, we got a conclusion that the average annual percent change (AAPC) of the standardized mortality rate in urban areas was $2.8 \%(P<0.05)$, whereas in rural areas it was $6.5 \%$ $(P<0.05)$ (Figure 2 and Table 3$)$.

Age-specific and Gender characteristics of lung cancer mortality

Lung cancer mortality rate increased with age, and was relatively lower before 45-50 years old, then increased dramatically after 50 years old and finally reached a peak after 85 years old. In sexes, lung cancer mortality also increased with age and peaked in the 85 age group. In urban areas, it peaked in the 85-year age group, reaching 717.90 per 100,000 men and 313.91 per 100,000 women. In rural areas, the mortality rate in men peaked at 527.43 per 100, 000 in the 85-year-old age group, whereas in women, the rate peaked at 237.46 per 100,000 in those aged 85 (Figure 3).

Geographical distribution of lung cancer mortality

The trend surface analysis based on four aspects,

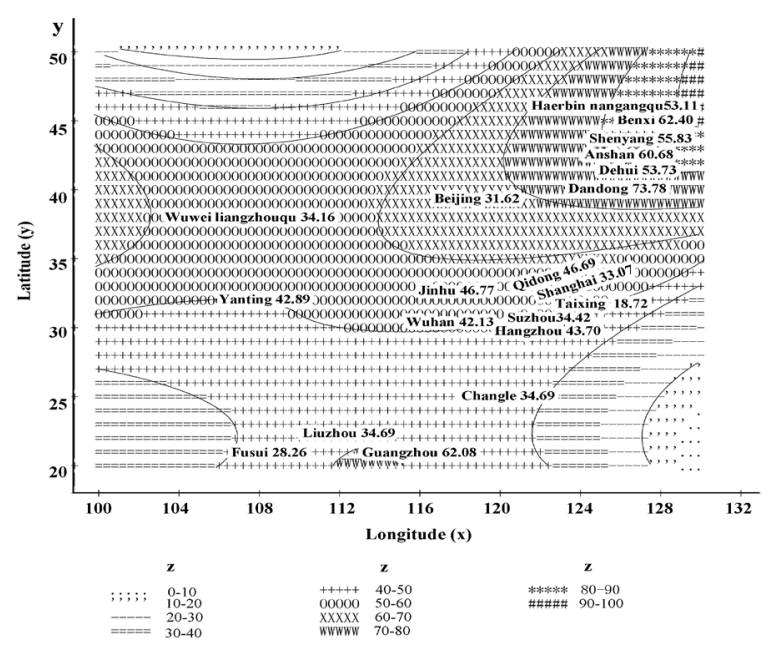

Figure 4. Geographical Distribution of the Standardized Mortality Rate of Male Lung Cancer in China

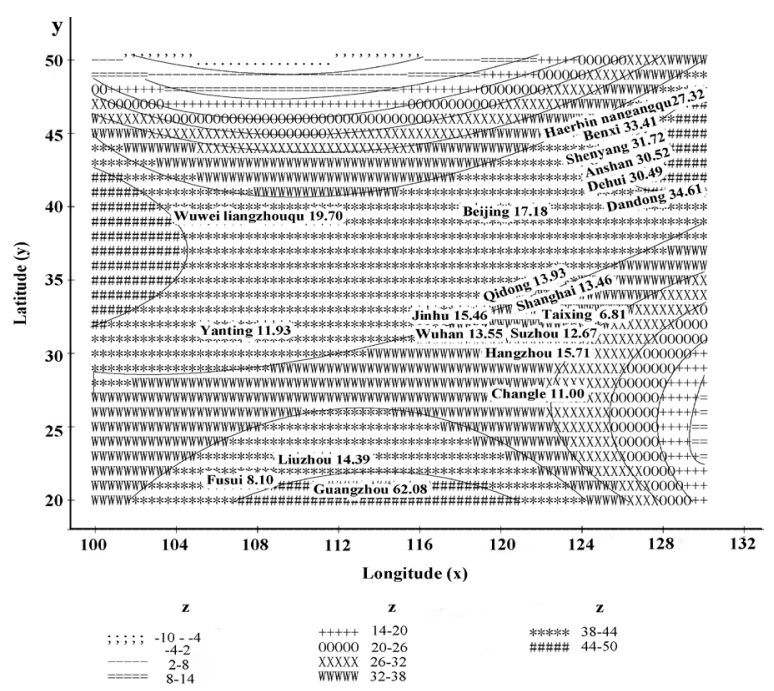

Figure 5. Geographical Distribution of the Standardized Mortality Rate of Female Lung Cancer in China 
Table 1. Mortality for Lung Cancer, by 4 Models, in China from 1991 to $2013\left(1 / 10^{5}\right)$

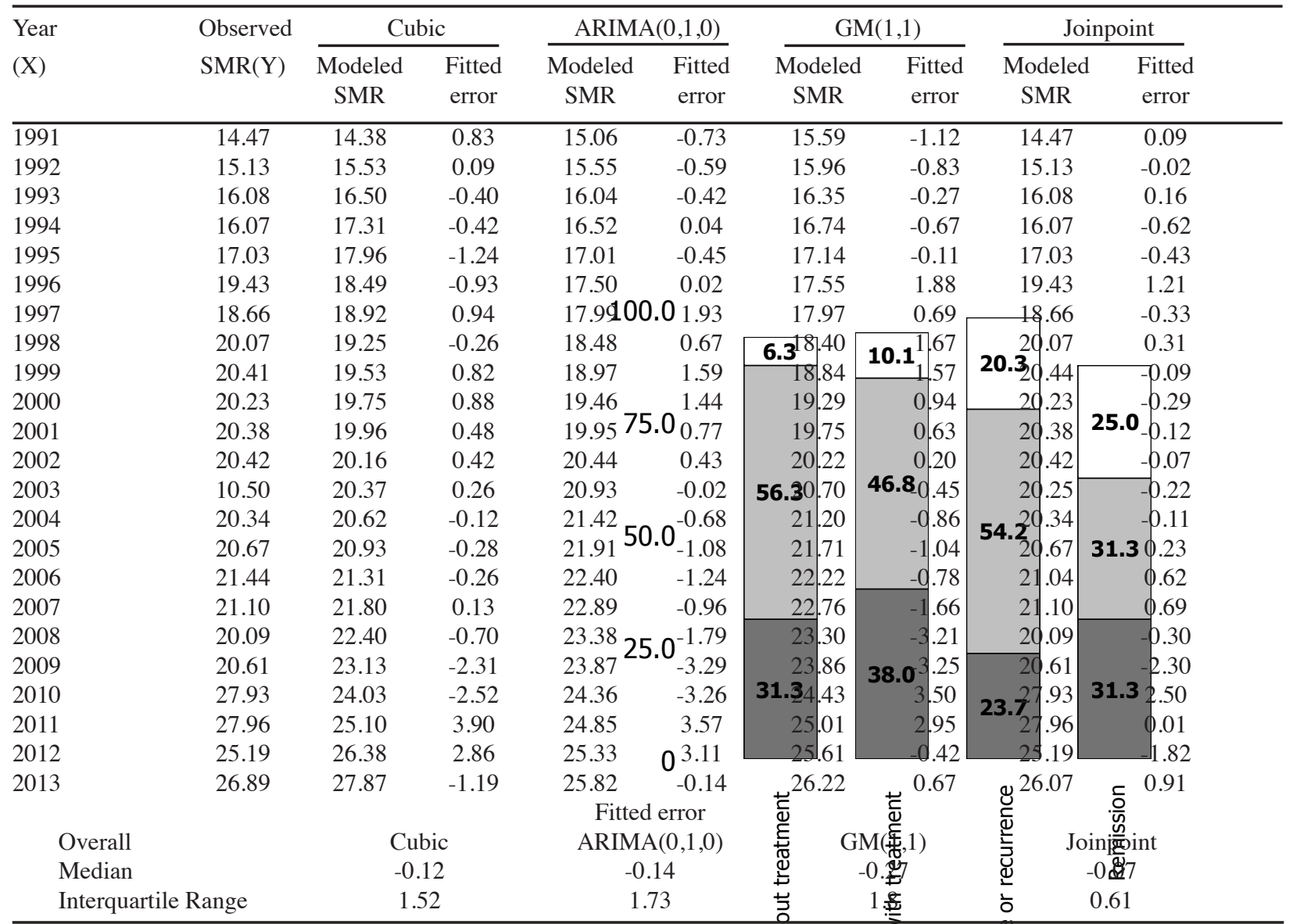

*SMR-standardized mortality rates

Table 2. Predicted Mortalities for Lung Cancer, by 4 models, in China During 2014-2018(1/10 $)$

\begin{tabular}{lcccccc}
\hline Year & Cubic & $\begin{array}{c}\text { ARIMA } \\
(0,1,0)\end{array}$ & $\begin{array}{c}\text { GM } \\
(1,1)\end{array}$ & Joinpoint & \multicolumn{2}{c}{ Overall } \\
\hline 2014 & 29.60 & 26.31 & 26.85 & 29.72 & $28.12 \pm 1.79$ & $(25.27,30.97)$ \\
2015 & 31.59 & 26.80 & 27.49 & 31.14 & $29.26 \pm 2.46$ & $(25.34,33.17)$ \\
2016 & 33.86 & 27.29 & 28.15 & 33.99 & $30.82 \pm 3.60$ & $(25.09,36.55)$ \\
2017 & 36.43 & 27.78 & 28.82 & 35.42 & $32.11 \pm 4.44$ & $(25.04,39.18)$ \\
2018 & 39.33 & 28.27 & 29.51 & 36.84 & $33.49 \pm 5.43$ & $(24.85,42.13)$ \\
$\bar{x} \pm$ SD & $34.16 \pm 3.85$ & $27.29 \pm 0.77$ & $28.16 \pm 1.05$ & $23.42 \pm 2.95$ & & \\
$95 \%$ CI & $(29.38,38.94)$ & $(26.33,28.25)$ & $(26.86,29.47)$ & $(29.75,37.09)$ & & \\
\hline
\end{tabular}

*SD-standard deviation; CI-confidence interval

namely the R-square test, F-test, goodness-of-fit order by order test, and subjective judgment method, was built. A meaningful third-order trend surface equation for lung cancer mortality trends in men $(\mathrm{F}=2.59$, $P<0.0195, \mathrm{R} 2=0.3802)$ and in women $(\mathrm{F}=6.15, P<0.0001$, $\mathrm{R} 2=0.6485)$ of the geographic distribution can be predicted using $\mathrm{z}(1)$ and $\mathrm{z}(2)$ :

$z(1)=1193.0800-113.5692 * x+274.9507 * y+1.8012 * x * x-$ $5.4026 * x * y+0.9535^{*} y^{*} y-0.0075 * x * x * x+0.0218^{*} x^{*} x * y+$ $0.0083 * x * y * y-0.02059 * y^{*} y^{*} y$

$z(2)=1363.34-83.7001 * x+164.1833 * y+1.2301 * x * x-$ $3.4262 * x^{*} y+0.9168^{*} y^{*} y-0.0049 * x^{*} x^{*} x+0.01315^{*} x^{*} x^{*} y+$ $0.0075 * x * y * y-0.0185 * y^{*} y^{*} y$

In the two third-order trend surface equation, $\mathrm{x}$ stands for longitude of the cities/towns, y stands for latitude, and $\mathrm{z}$ stands for the standardized mortality ratio of lung cancer in various areas. These two models can fit goodness as $38.02 \%$ (male) and $64.85 \%$ (female) of lung cancer mortality rates in China. Lung cancer mortality rates have a certain geographical distribution in different genders and rates were higher in men than that in women. It appeared to be related to the geographical distribution: At the provincial level, it was highest in Northeastern China (such as Liaoning, Jilin, Heilongjiang), followed by northern China (such as Hebei, Beijing) and the coastal provinces in eastern China (Shanghai, Jiangsu, Zhejiang), and it gradually decreased as the location went southwestwards or northwestwards. At the county level, Dandong, Anshan, Shenyang, Benxi of Liaoning province, Dehui of Jilin province, and Harebin nangangqu of Heilongjiang province stood out with the highest rates of lung cancer mortality. It is worth noting that the mortality in Guangzhou, a southern city, is also very high (Figure 4 and 5). 
Characteristics and Prediction of Lung Cancer Mortality in China from 1991 to 2013

Table 3. APC and AAPC ${ }^{\text {b }}$ of Age-standardized Rates for Lung Cancer in China from 1991-2013

\begin{tabular}{lcccc}
\hline Trend & APC $^{\text {a }}$ AAPC & Urban & Rural & Overall \\
\hline Segment 1 & Years & $1991-2005$ & $1991-2000$ & $1991-2001$ \\
& APC $(95 \% \mathrm{CI})$ & $1.0^{\mathrm{c}}(0.1,1.9)$ & $4.5^{\mathrm{c}}(2.7,6.3)$ & $3.4^{\mathrm{c}}(1.8,4.9)$ \\
Segment 2 & Years & $2005-2008$ & $2000-2003$ & $2001-2004$ \\
& APC $(95 \% \mathrm{CI})$ & $-2.8(-5.6,38.2)$ & $18.2(-1.7,42.1)$ & $25.1^{\mathrm{c}}(3.3,51.5)$ \\
Segment 3 & Years & $2008-2013$ & $2003-2006$ & $2004-2013$ \\
& APC $(95 \% \mathrm{CI})$ & $16.3(-5.3,3.2)$ & $-0.4(-17.2,2.8)$ & $4.6^{\mathrm{c}}(2.8,6.4)$ \\
Segment 4 & Years & $\mathrm{ND}^{\mathrm{d}}$ & $2006-2013$ & $\mathrm{ND}^{\mathrm{d}}$ \\
& APC $(95 \% \mathrm{CI})$ & $\mathrm{ND}^{\mathrm{d}}$ & $6.4^{\mathrm{c}}(3.8,9.0)$ & $\mathrm{ND}^{\mathrm{d}}$ \\
Full Range & Years & $1991-2013$ & $1991-2013$ & $1991-2013$ \\
& AAPC $(95 \% \mathrm{CI})$ & $2.8^{\mathrm{c}}(2.1,3.5)$ & $6.5^{\mathrm{c}}(5.5,7.6)$ & $7.7^{\mathrm{c}}(6.7,8.8)$ \\
\hline
\end{tabular}

*APC -annual percent change; AAPC $^{\mathrm{b}}$-annual percent change; ${ }^{\mathrm{c}}$-significantly different from zero at alpha=0.05 $(P<0.05)$; ND ${ }^{\mathrm{d}}$-no data

\section{Estimation and forecasting}

The up-to-date dates on mortality in China are those from the continuing surveillance system and are usually in small sample sets, and it may not be fully used and it is risky to assume the derived distribution. Consequently, we applied four independent but complementary predictive models --- curve estimation, time series model, gray model, Joinpoint regression for data analysis to find a suitable trend for mortality of lung cancer in the next five years. We assumed that the factors such as social, economic, environmental and lifestyle aspects of the Chinese population had no dynamical change. This made previous data of lung cancer mortality from 1991-2013 suitable for building a predictive model. The error value (ERR) and the 95\% Confidence Interval (95\%CI) are used to be the forecasting accuracy measures.

Curve estimation: The cubic curve model gave acceptable predictive estimates of mortality and was stable (R2 $=0.87, \mathrm{~F}=44.18, P<0.001)$ (Tables 1 and 2). The equation is: $\mathrm{y}=11.4210+1.7130 * \mathrm{x}-0.1240 * \mathrm{x} * \mathrm{x}+0.003 *$ $\mathrm{x}^{*} \mathrm{x} * \mathrm{x}$, where $\mathrm{x}$ stands for the year, and $\mathrm{y}$ stands for mortality rate.

Time series model: For the final ARIMA model, we found that the ARIMA $(0,1,0)$ model was the most suitable for prediction $(\mathrm{R} 2=0.76, P=0.77)$ (Tables 1 and 2$)$.

Gray model $(\mathrm{GM})$ : GM $(1,1)$ models were used to perform the forecast, with fitting parameters: $a=-0.02$ $\mathrm{b}=15.08$. The equation is: $\mathrm{X}(\mathrm{t}+1)=652.000 .02 \mathrm{t}-638.16$ $(\mathrm{C}=0.43, P=0.83$ ) (Tables 1 and 2$)$.

Joinpoint regression: The Joinpoint regression model was used to provide estimated annual percentage change (AAPC) and to detect points in time where a statistically significant change over time in linear slope of the trend occurred. In China, the estimated average annual percent change (AAPC) of the standardized mortality rate was 7.7\% during1991-2013 (Tables 1, 2 and 3).

\section{Discussion}

Lung cancer is one of the five leading tumors. The standardized mortality rate SMR of lung cancer in China has been increasing steadily in both males and females since the $1970 \mathrm{~s}$, and according to our four forecast analysis the upward trend will continue to grow in the future 5 years. High mortality of lung cancer has naturally made it to be a key issue to the health organizations all over the world and will pose a serious threat to human health.
China is an industrialized country, and lung cancer will be the leading killer of all cancers due to modern social customs and insalubrious lifestyles. Therefore, etiology should be explored and the prevention and management should be focused on to reduce the risk factors. The known factors for lung cancer include smoking, air pollution, and certain types of occupational exposure and the significance of each of these factors appears to vary with sex, age, and region.

Accumulated data has shown that mortality rates of lung cancer appear to be a relatively large difference between urban and rural areas. The mortality rates in urban areas are higher than those in rural areas. Because of lower income in rural areas, biomass (for example, crop residues and wood) has been the dominant household fuel, while in cities the use of coal has been more common (Lan et al., 2002). Previous studies have indicated that the relationship between wood smoke and lung cancer has been weaker than that between coal and lung cancer in epidemiological studies (Bruce et al., 2000). Exposure to urban air pollutants including indoor air pollution (biomass and coal use) and outdoor air pollution (chemical releases from industry and a growing transportation sector) is associated with lung cancer (Pope III et al., 2002). These suggest that there is an emergency request for continued development of national programs on smoking cessation, and it is a long-term challenge to improve air quality in China.

Lung cancer mortality rate increased with age and increased rapidly in people over the age of 45-50, and peaked in the 85 age group. Tobacco consumption and environmental pollution are the major risk factors. People who have a history of smoking over 45 year-old require regular medical examination every year. Lung cancer mortality rate was higher in men than that in women. The reasons for higher rates of lung cancer in males may relate to sex-specific differences in exposure to risk factors. More specifically, major differences exist between males and females with regard to lung cancer risk factors: In males, cigarette smoking and occupational exposure play important roles, whereas in females, indoor air pollution, derived from cooking fuel and household coal consumption, are more important risk factors (Du et al., 1996).

A trend-surface analysis procedure was used to identify the geographical distribution of lung cancer. The trend surface analysis indicated that there was a tendency for 
lung mortality rates to be higher in the northeastern and the coastal provinces rather than the central or western areas. Due to more smokers, longer heating time and centralized mining, high risk areas are mainly concentrated in northern China, such as Dandong, Anshan, Shenyang, Benxi of Liaoning province, Dehui of Jilin province, and Harebin nangangqu of Heilongjiang province. Lung cancer has regional features similar to liver carcinoma. Both of the cancers are concentrated on the coastal provinces in eastern China. However, lung cancer has a relatively low mortality rate in the northeast, while the mortality of lung cancer in northeastern China, such as Liaoning, Jilin and Harbin, is very high. Comprehensive prevention and control measures of lung cancer should be channeled to the northeast areas and the coastal provinces in eastern China (Fang et al., 2015).

In conclusion, the mortality rate of lung cancer is increasing and will continue to rise steadily in the foreseeable future and imposing an enormous burden on patients, health-care professions, and society. Short- and long-term lung cancer prevention strategies should be focused on reducing the mortality of lung cancer. For the general population, effective measures should focus on health education to increase public awareness, and early detection and prevention of lung cancer. For the Chinese government, further efforts should be made on specific and timely interventions measures to reduce smoking rates and air pollution in China. What is more, greater attention to standardizing the clinical management of lung cancer is required, helping patients with lung cancer to maintain a good quality of life.

\section{Acknowledgements}

The study was supported by grants from the Tumor Research Center of Collaboration and Innovation, STUMC the Guangdong Provincial Key Laboratory of Infectious Diseases and Molecular Immunopathology, China and National Science Foundation of China (NO.81470152).

\section{References}

Bruce N, Perez-Padilla R, Albalak R (2000). Indoor air pollution in developing countries: a major environmental and public health challenge. Bull World Health Organ, 78, 1078-92.

Chen JG, Zhang SW (2011). Liver cancer epidemic in China: past, present and future. Semin Cancer Biol, 21, 59-69.

Chen W, Zhang S, Zheng R, et al (2013). Report of cancer incidence and mortality in China, 2009. China Cancer, 22, 2-12.

Chen W, Zheng R, Zhang S, et al (2014). Report of cancer incidence and mortality in China, 2010. China Cancer, 23, 1-10.

Chen W, Zheng R, Zhang S, et al (2015). Report of cancer incidence and mortality in China, 2011. China Cancer, 24, 1-10.

Fang J-Y, Wu K-S, et al (2015). Mortality Characteristics and Prediction of Liver Cancer Mortality in China from 1991 to 2012. Asian Pac J Cancer Prev, 16, 1959-64.

Chen Z (2008). The Third National Mortality Retrospective Sampling Survey Report. Beijing: Peking Union Medical College Publication House.
China health statistics yearbook (2005). Beijing: Peking Union Medical College Publication House.

China health statistics yearbook (2006). Beijing: Peking Union Medical College Publication House.

China health statistics yearbook (2007). Beijng: Peking Union Medical College Publication House.

China health statistics yearbook (2008). Beijing: Peking Union Medical College Publication House.

China health statistics yearbook (2014). Beijing: Peking Union Medical College Publication House.

Du Y-x, Cha Q, Chen X-w, et al (1996). An epidemiological study of risk factors for lung cancer in Guangzhou, China. Lung Cancer, 14, 9-37.

He J, Chen W (2011). Chinese cancer registry annual report. Beijing: Military Medical Science Press,

He J, Chen W (2012). Chinese cancer registry annual report. Beijing: Military Medical Science Press,

Huang S, Wang W, Zeng C, et al (2013). Application of Gray Metabolic GM $(1,1)$ Model in Prediction of Annual Total Yields of Chinese Aquatic Products. Asian Agricultural Research, 5, 21-25.

Lan Q, Chapman RS, Schreinemachers DM, et al (2002). Household stove improvement and risk of lung cancer in Xuanwei, China. J National Cancer Institute, 94, 826-35.

Li Q, Guo N-N, Han Z-Y, et al (2012). Application of an autoregressive integrated moving average model for predicting the incidence of hemorrhagic fever with renal syndrome. Am J Tropical Med Hygiene, 87, 364-70.

Pope III CA, Burnett RT, Thun MJ, et al (2002). Lung cancer, cardiopulmonary mortality, and long-term exposure to fine particulate air pollution. JAMA, 287, 1132-41.

Qiu D, Katanoda K, Marugame T, et al (2009). A Joinpoint regression analysis of long term trends in cancer mortality in Japan (1958-2004). Int J Cancer, 124, 443-8.

Shi X-J, Au WW, Wu K-S, et al (2014). Mortality characteristics and prediction of female breast cancer in China from 1991 to 2011. Asian Pac J Cancer Prev, 15, 2785-91.

Spaeder M, Fackler J (2012). A multi-tiered time-series modelling approach to forecasting respiratory syncytial virus incidence at the local level. Epidemiol Infect, 140, 602-7.

Tang W-R, Fang J-Y, Wu K-S, et al (2014). Epidemiological characteristics and prediction of esophageal cancer mortality in China from 1991 to 2012. Asian Pac J Cancer Prev, 15, 6929-34.

Wei K, Liang Z, Liu J, et al (2012). History of cancer registration in China. Zhonghua yi shi za zhi, 42, 21-5 (in Chinese).

World Health Organization (2012). Cancer. Fact sheet no 297. Available at http://www.who.int/mediacentre/ factsheets/ fs297/en/. Accessed September 23, 2012.

Zhang X, Zhang T, Young AA, et al (2014). Applications and comparisons of four time series models in epidemiological surveillance data. PloS One, $9,88075$.

Zhao P, Chen Wq (2008). Chinese Cancer Registry Annual Report. Beijing: Military Medical Science Press.

Zhao P, Chen W (2009). Chinese Cancer Registry Annual Report. Beijing: Military Medical Science Press.

Zhao P, Chen W (2010). Chinese Cancer Registry Annual Report. Beijing: Military Medical Science Press. 